
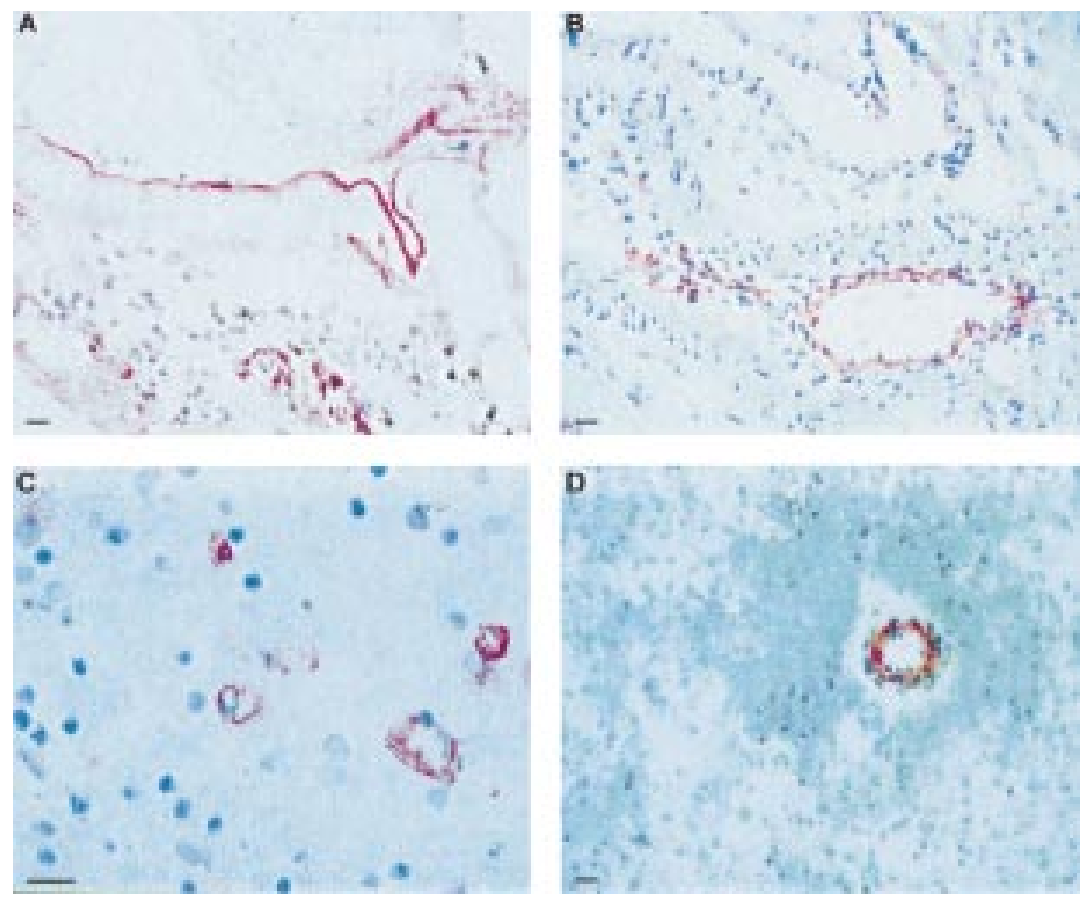

Immunostaining with the TN-11 Ab fragment or the BC-1 mAb shows the presence of the type III repeat $C T N-(A)$ and $E D-B+F N-(B)$ isoforms in angiomatous vessels. These isoforms are also present in the wall of vessels of the cerebral tissue adjacent to the angiomatous nidus (TN: $C ; F N: D)$. Bar $=10 \mu \mathrm{m}$.

Previous findings showed that ED-B+FN presents with conformational modifications in its central part and results from deregulation of FN pre-mRNA. ${ }^{4}$ The distribution of this isoform was found to be highly restricted in normal adult tissues. By contrast, ED-B+ FN exhibited widespread distribution in the vasculature of fetal tissues, including brain, and of several types of malignancies. It was therefore regarded as a marker of angiogenesis. ${ }^{5}$

Similarly, the type III repeat C TN isoform, recognised by the $\mathrm{Ab}$ fragment TN-11, was found to occur in the vascular walls of anaplastic gliomas. Northern blot analysis showed that the mRNA of this isoform was undetectable in normal tissues and some malignancies, but was present in large amounts in fetal tissues, including brain, and in glioblastomas ${ }^{3}$

Recent advances in the pathology of cerebral AVMs suggest that these lesions might not be static. Tyrosine kinase, an endothelial cell specific receptor upregulated in glioblastomas, was found to be highly expressed in both AVMs and in the vessels of cerebral tissue bordering the malformations, by contrast with the down regulation occurring in the vasculature of the normal brain. ${ }^{1}$ The pattern of distribution of structural proteins was consistent with the hypothesis of diffuse activation of angiogenesis, without specific relation to individual vessel types. ${ }^{2}$

Furthermore, use of the cell proliferation marker MIB-1 showed endothelial proliferation in arterioles, venules, and capillaries of the cerebral tissue neighbouring AVMs.

The present findings indicate that a particular FN isoform, mainly expressed by the vasculature of fetal and tumorous tissues, as well as a TN isoform typically detected in the walls of vessels in anaplastic gliomas, also occur in AVMs and in vessels of adjacent cerebral tissue, but that both isoforms are absent in normal brain. This evidence provides further support to the hypothesis of ongoing angiogenesis in and around these lesions.
The presence of angiogenic features in AVMs might result from maintenance of proliferating and remodelling potentials, or from vascular structures subjected to increased blood flow and pressure. Occurrence of these features also in vessels lying in areas peripheral to the nidus might be related to recruitment of the neighbouring vasculature, possibly dependent on focal ischaemia in the setting of arteriovenous shunting. ${ }^{12}$ However, the presence in apparently normal vasculature of molecules typically occurring in fetal tissues and malignancies indicate that cerebral AVMs may not be static lesions. Further studies are needed to ascertain whether this phenomenon results merely from haemodynamic stress or actually reflects an intrinsic growth potential. Should this second be the case, current therapeutic strategies would possibly require revision.

This study was partially supported by the National Research Council (CNR), AIRC and the Ministry of University and Scientific Research (MURST). We thank Sergio Deseri, EE, for his technical help and Mr. Thomas Wiley for manuscript revision.

ANTONIO PAU

A DORCARATTO

G L VIALE

DI S C A T Departement of Surgery, Division of Neurosurgery, University of Genoa Medical School, S Martino Hospital, Pad 2, Largo Rosanna Benzi 10,

16132 Genova, Italy

P CASTELLANI

A SIRI

L ZARDI

Laboratory of Cell Biology National Cancer Institute, Genoa, Italy

Correspondence to: Dr A Pau

1 Hatva E, Jääskeläinen J, Hirvonen $\mathrm{H}$, et al. Tie endothelial cell-specific receptor tyrosine kinase is upregulated in the vasculature of arternase is upregulated in the vasculature of arterovenous malformations.
Neurol 1996;55:1124-33. a specific response to haemodynamic stress in
2 Rothbart D, Awad IA, Jiyon L, et al. Expression of angiogenic factors and structural proteins in central nervous system vascular mal
tions. Neurosurgery 1996;38:915-25.

3 Carnemolla B, Castellani P, Borsi L, et al. Identification of a glioblastoma associated tn-c isoform by a high affinity recombinant antibody. Am F Pathol 1999;154:1345-52.

4 Carnemolla B, Leprini A, Allemanni G, et al. The Inclusion of type III repeat ED-B in the fibronectin molecule generates conformational modifications that unmask a cryptic sequence. f Biol Chem 1992;267:24689-92.

5 Castellani P, Viale G, Dorcaratto A, et al. The fibronectin isoform containing the ED-B oncofetal domain: a marker of angiogenesis. Int $\mathcal{f}$ Cancer 1994;59:612-18.

Hashimoto's encephalopathy presenting as "myxoedematous madness"

The neuropsychiatric sequelae of hypothyroidism range from lethargy and mental slowing to the florid psychotic illness referred to as "myxoedematous madness". The last condition is characterised by frank hypothyroidism accompanied by psychosis, and may respond completely to thyroxine. ${ }^{1}$ More recently described is a syndrome of subacute encephalopathy, associated with high titres of thyroid autoantibodies, raised CSF protein, EEG abnormalities, and perfusion deficits in the presence of normal structural neuroimaging. ${ }^{2-4}$ In most cases, the encephalopathy occurs without any gross change in circulating concentrations of thyroid hormones, suggesting that an inflammatory process is responsible for the cerebral dysfunction. In the absence of pathological data, the evidence for a specific pathogenetic mechanism is largely circumstantial: a small vessel vasculitis and immune complex deposition have both been suggested. ${ }^{34}$

Although none of the published cases of Hashimoto's encephalopathy has described psychosis as a primary feature, it is possible that "myxoedematous madness", a condition first described in detail by Asher in $1949^{1}$ lies in a range of encephalopathic phenomena mediated by autoimmune mechanisms. This suggestion would certainly be consistent with the range of clinical presentations of other autoimmune cerebral vasculitides. ${ }^{5}$ As autoimmune thyroiditis is the commonest cause of thyroid failure in this country, ${ }^{6}$ it is likely to have been present in at least some of Asher's original 14 cases. Although most had florid myxoedematous features at psychiatric presentation, this may simply reflect the difficulty of diagnosing subclinical thyroid disease before rapid laboratory assays became widely available. Many features of the present case, however, favoured an endocrine rather than an inflammatory mechanism, suggesting that the condition of "myxoedematous madness", though rare, remains a valid diagnostic entity.

A 63 year old market stallholder without medical or psychiatric history was brought to a local psychiatric hospital by the police. His business had been in decline for several months, and his family had noticed uncharacteristic emotional lability. In the weeks preceding admission he had experienced delusions and hallucinations, and exhibited uncharacteristic behaviour. He had reported a vision of the crucifixion, and hearing the voice of his dead mother. He claimed that his house was occupied by the devil, drove around aimlessly in his car, and appeared constantly fearful and withdrawn. On the day of admission he had made a bonfire in the garden and burned his wife's clothes, family photographs, furniture, and business papers. When his wife and son tried to intervene he 
Table 1 Laboratory and neuropsychological results at presentation (A) and at 12 month follow up (B)

\begin{tabular}{lll}
\hline Laboratory (units) & $A$ & $B$ \\
\hline Full blood count & Normal & Normal \\
Erythocyte sedimentation rate & 12 & 6 \\
Urea and electrolytes & Normal & Normal \\
Liver function tests & Normal & Normal \\
Antinuclear antibody & Negative & Negative \\
B12 and folate & Normal & Not tested \\
VDRL & Negative & Not tested \\
Thyroid stimulating hormone (mU/1) & 58.4 & 0.87 \\
Free T4 (pmol/1) & 7.4 & Not tested \\
Antithyroid microsomal antibody titres & $1: 25600$ & $1: 1600$ \\
Psychometric (normal/predicted range): & & \\
Folstein MMSE $(>24)$ & $25 / 30$ & 25 \\
NART IQ & 10 th percentile & 18 th percentile \\
WAIS-R (verbal) & 13 th percentile & Not tested \\
WAIS-R (performance) & 27 th percentile & Not tested \\
FAS verbal fluency $(>30)$ & 25 & 23 \\
Cognitive estimates test $(<6)$ & 10 & 11 \\
Graded naming test $(>15)$ & $10 / 30$ & $16 / 30$ \\
Digit span forwards $(>5)$ & 7 & 6 \\
Rey-Osterreith complex figure (copy) $(36)$ & 25.5 & 24 \\
Rey-Osterreith complex figure (recall) $(30 \%)$ & Not tested & $75 \%$ \\
\hline
\end{tabular}

became aggressive and threatened them with a saw. The general practitioner was called and suspected either an acute psychosis or a severe depressive illness. Police assistance was requested because of the patient's continuing violent behaviour.

On admission he was unkempt but cooperative and apparently euthymic. He denied depression, but displayed no insight into the irregularity of his behaviour. No psychotic features were seen, although during the admission he consistently rationalised all reported psychotic phenomena. $\mathrm{He}$ was aggressive towards staff and made repeated attempts to abscond. General physical examination was unremarkable. Neurological examination was normal except for spoken language, which was fluent and grammatical, but contained word finding pauses, circumlocutions, and occasional semantic errors (for example, "I just want to get my feet back on the table"). Formal neuropsychological testing, and a screen of laboratory tests for reversible causes of encephalopathy, were performed on admission, and results are presented below (column A). Attention is drawn to his mild naming deficit, and poor performance on the Rey figure, which was due to planning rather than visuospatial errors, suggesting a predominantly "dysexecutive" pattern. CT and EEG were both normal, and SPECT disclosed widespread but mild cortical hypoperfusion. Trifluoperazine ( $2 \mathrm{mg}$ twice daily) was started on admission, and thyroxine ( $75 \mu \mathrm{g}$ once daily) added 1 week later. His mental state and behaviour stabilised, leading to discharge after 2 months.

At 6 month follow up the patient had stopped neuroleptic drugs, but continued taking thyroxine. He reported feeling "back to normal", had bought a new house, and was working as a part time shop assistant. $\mathrm{He}$ still had subtle word finding difficulties, and was referred to the regional memory clinic for further evaluation, which took place 6 months later. Behavioural assessment showed persisting deficits in delayed recall of verbal material, verbal fluency, and visuospatial function. Formal psychometric testing, blood tests, and SPECT were repeated, 1 year after the original examinations. Laboratory and neuropsychological results are presented in the table. It is of note that, whereas his naming ability had improved, performance on frontal executive tasks remained impaired The appearance of the follow up SPECT dif- fered minimally, if at all, from the first examination.

In summary, therefore, this patient presented in clear consciousness with a first subtle executive and linguistic neuropsychological disturbance, on the background of gradual behavioural and affective change. He was profoundly hypothyroid due to an autoimmune thyroiditis, but there was no clinical evidence of thyroid failure other than the abnormal mental state. The psychiatric component of his illness recovered fully, and the antithyroid microsomal antibody titre fell markedly after thyroxine replacement, alremained unchanged. Corticosteroids were not used at any stage.

The response to thyroxine does not, in itself, imply that the cerebral illness had an endocrine origin; a recent report described a patient with a subacute encephalopathic illness and compensated hypothyroidism in the presence of increased antimicrosomal antibodies, all of which responded to thyroxine replacement alone. ${ }^{4}$ In that case, however, both EEG and SPECT were abnormal, the SPECT showing multiple areas of severely reduced perfusion, which normalised with treatment. By contrast, in the present case the EEG was normal and the SPECT abnormality was marginal and changed little, if at all, with treatment. The evidence for a significant vasculitic component to the illness is, therefore, unconvincing

The mild and relatively circumscribed neuropsychological deficits coupled with florid psychotic phenomena, also contrast with the profound global disturbance of cognition usually associated with Hashimoto's encephalopathy. ${ }^{3}$ This distinction suggests that microvascular disruption and thyroid hormone depletion may emphasise different aspects of the clinical range in Hashimoto's encephalopathy. Although the present case would support Asher's conclusion that the psychiatric features of Hashimoto's encephalitis typically respond to thyroid replacement, it additionally suggests that subtle neuropsychological deficits may be apparent even in the absence of obvious cerebral perfusion deficits, and that these may not be fully reversible.

P GARRARD

J R HODGES

University of Cambridge Neurology unit, Addenbrooke's Hospital, Cambridge CB2 2QQ, UK episode of acute psychosis, and evidence of though his mild neuropsychological deficits
P J DE VRIES N HUNT University of Cambridge Department of Psychiatry, Addenbrooke's Hospital, Cambridge CB2 2QQ, UK

A CRAWFORD

J R HODGES

MRC Cognition and Brain Sciences Unit, 15 Chaucer Road, Cambridge CB2 2EF, UK

K BALAN

Department of Nuclear Medicine, Addenbrooke's Hospital, Cambridge CB2 2QQ, UK

Correspondence to: Dr P Garrard, University of Cambridge Neurology Unit, Box 165, Addenbrooke's Hospital, Cambridge CB2 2QQ, UK email garrard@cnbc.cmu.edu

1 Asher R. Myxoedematous madness. BMF 1949;555-62

2 Thrush DC, Boddie HG. Episodic encephalopathy associated with thyroid disorders. $f$ 3 Shaw PJ, Walls TJ, Newman PK, et al. Hashimoto's encephalopathy: a steroid responHashimoto's encephalopathy: a steroid respon-
sive disorder associated with high anti-thyroid sive disorder associated with high anti-thyroid
antibody titers: report of 5 cases. Neurology 1991;41:228-33.

4 Forchetti CM, Katsamakis G, Garron DC. Autoimmune thyroiditis and a rapidly progressive dementia: global hypoperfusion on SPECT scanning suggests a possible mechanism. Neurology 1997;49:623-6.

5 Scolding NJ, Jayne DR, Zajicek J, et al. Cerebral vasculitis: recognition, diagnosis and management. $Q \mathcal{F}$ Med 1997;90:61-73.

6 Dayan CM, Daniels GH. Chronic autoimmune thyroiditis. N Engl F Med 1996;335:99-107.

\section{Alien hand sign in Creutzfeldt-Jakob} disease

The clinical picture of Creutzfeldt-Jakob disease (CJD) includes various movement disorders such as myoclonus, parkinsonism, hemiballism, and dystonia. We report on a patient with CJD who manifested the alien hand sign. We suggest that CJD should be included in the differential diagnosis of diseases which present with an alien hand.

Creutztfeldt-Jakob disease, one of the human prion diseases, is characterised by rapidly progressive mental and motor deterioration. ${ }^{1}$ Involuntary movements occur in above $90 \%$ of the patients in the course of the disease, the most common being myoclonus. ${ }^{1}$ Other movement disorders range from tremor to chorea, athetosis, dystonia, and hemiballism. ${ }^{1}$ We report on a patient with CJD who presented with an alien hand.

Alien hand is a rare and striking phenomenon defined as "a patient's failure to recognise the action of one of his hands as his own". ${ }^{2}$ One of the patient's hands acts as a stranger to the body and is uncooperative. Thus, there is loss of feeling of ownership but not loss of sensation in the affected hand. Originally described in callosal tumours, ${ }^{3}$ the aetiology of alien hand also includes surgical callosotomy, ${ }^{4}$ infarction of the medial frontal cortex, occipitotemporal lobe, and thalamus, ${ }^{15}$ infection, ${ }^{6}$ and corticobasal degeneration. ${ }^{5}$

A 70 year old, right handed Jewish man born in Argentina, living in Israel for the past 20 years, was admitted to the Neurology Department. Until a month before his admission, he was apparently healthy and helped in the accounting office of the village where he lived. His neurological illness had presented insidiously during the past month with unsteadiness of gait and frequent falls. $\mathrm{He}$ also manifested behavioural changes, became aggressive, and had visual hallucinations, perceiving insects and mice moving through his visual field. Often, he expressed his fear from seeing that the "ceiling was 